\title{
Creación de un plan para aumentar los índices de adopción digital en los canales de una institución financiera
}

\author{
Carlos David De La Ossa Paternina* \\ Recibido: 2 de agosto de 2020 \\ Revisado: 4 de septiembre de 2020 \\ Aprobado: 5 de octubre de 2020
}

Citar como:

De La Ossa Paternina, C. D. (2021). Creación de un plan para aumentar los índices de adopción digital en los canales de una institución financiera. Revista CIFE, 23(38). https://doi.org/10.15332/22484914.6132

\section{Resumen}

Hay una tendencia mundial a la digitalización de servicios, donde el sector financiero es uno de los que está teniendo mayores transformaciones. El surgimiento de las fintech ha aportado, en gran medida, a la evolución de las soluciones que pueden brindarse mediante el uso de un celular, lo que resulta imprescindible para garantizar la permanencia en el mercado en unos años. En este orden de ideas, la banca móvil se convierte en una necesidad y no en una elección. Este trabajo ofrece una solución que impulsa la adopción de los desarrollos y cambios tecnológicos implementados en la entidad financiera Banco Amigo. Para ello, en primera instancia, se realiza una investigación a través de encuestas y de un modelo analítico para identificar las barreras y limitaciones de los clientes que no hacen uso de los canales digitales. La segunda parte se enfoca en la creación de estrategias en pro de acompañar a los clientes para que adopten el uso de estos canales, con el fin de aumentar el porcentaje de usuarios que ingresan y hacen sus transacciones por estos medios.

Palabras clave: adopción digital, canales digitales, banca móvil, transformación digital y sector financiero.

Clasificación JEL: G14, G21, O31, O33, P34.

\footnotetext{
* Universidad del Rosario. ORCID: https://orcid.org/0000-0002-9959-5707. Correo electrónico: carlosdavi.delaossa@urosario.edu.co
} 


\title{
CIIFF 38
}

\section{Creating a plan to increase digital adoption rates in a financial institution's channels}

\begin{abstract}
There is a worldwide trend towards digitization of services, being the financial sector one of those that is undergoing major transformations. In this sense, the rise of fintech has contributed, largely, to the evolution of solutions that can be provided through the use of a cell phone, which is essential to ensure the permanence in the market in a few years. In this order of ideas, mobile banking becomes a necessity and not a choice. This paper offers a solution that promotes the adoption of technological developments and changes implemented in the financial institution Banco Amigo. To do so, in the first place, a research is conducted through surveys and an analytical model to identify the barriers and limitations of clients who do not use digital channels. The second part focuses on the creation of strategies to support clients to adopt the use of these channels, in order to increase the percentage of users who enter and make their transactions by these means. Therefore, the handling of cash is reduced and all the transactional knowledge of clients is available to monitor their operations and proactively prevent fraud, thus increasing the bank's security and, consequently, improving user satisfaction. Moreover, it contributes to the reduction of money laundering and tax evasion, as well as the preservation of the environment.
\end{abstract}

Keywords: digital adoption, digital channels, mobile banking, digital transformation and financial sector.

JEL Classification System: G14, G21, O31, O33, P34.

\section{Introducción}

Según lo presentado por Schwab en el Foro Económico Mundial (2016), el mundo de los negocios comienza a cambiar para girar en torno a los nuevos modelos desarrollados por la dinámica que trae la cuarta revolución industrial. La economía de las próximas décadas estará basada en empresas cuyos procesos tendrán alta dependencia de la tecnología, por lo que muchos negocios tradicionales comienzan a tener una amenaza crítica de desaparecer, al ser reemplazados por nuevos fenómenos como Netflix, Uber o Rappi, que se adecúan a la flexibilidad tecnológica, el análisis de los datos y el aprovechamiento de la hiperconectividad de las personas a internet, a través del uso masivo de teléfonos inteligentes.

En el sector bancario la dinámica no es diferente; los modelos de negocio basados en el cliente para prestar servicios financieros están siendo altamente apalancados por la tecnología. El bajo costo del almacenamiento de datos, la fácil accesibilidad a la infraestructura a través de plataformas en la nube y el conocimiento de los usuarios que proporciona la analítica de información permiten crear emprendimientos alejados de la banca tradicional. Es así como nacen las fintech, que generan novedades en las opciones de financiación o movimientos de dinero a través de aplicaciones que el mercado ha comenzado a adoptar. 
La actual revolución tecnológica está modificando la forma de vivir, trabajar y relacionarse. Bajo estas circunstancias, las organizaciones están construyendo ecosistemas digitales que van de la mano con el nuevo comportamiento y necesidades que se vienen despertando en los seres humanos. Un claro ejemplo es la empresa de pagos digitales en China: WeChat, plataforma que entrega servicios financieros sin ser banco. Por medio de WeChat los usuarios pueden chatear, pero además pedir un taxi, comprar en supermercados o hacer transferencias de dinero a sus amigos, todo esto usando su celular (Heredia, 2019).

Lo anterior demuestra que ahora sobrevivirán aquellas empresas que tengan la capacidad de innovar para realizar su transformación digital y crear las estrategias adecuadas que le permitan a sus clientes adoptar estos procesos. El sector financiero ha pasado a ser uno de los más amenazados por la entrada de nuevos jugadores que no se tenían contemplados; Amazon, Google y la empresa china ya mencionada han creado modelos disruptivos que despiertan nuevos deseos en la sociedad y, a la vez, los suplen. Esto ha llevado a que los bancos tengan la obligación de reinventarse, desarrollar servicios digitales y promover su uso. El Banco Amigo no ha hecho caso omiso a estas tendencias, sino que ha volcado todos sus procesos a la transformación digital para potencializar sus ventas y relacionamiento con los clientes, creando productos y transacciones en los canales virtuales que logren dar soluciones inmediatas a las necesidades y deseos de los clientes en su día a día. Según el presidente del Banco Amigo, anualmente se vienen invirtiendo cerca de 300 millones de dólares en la implementación de la banca móvil y para el 2020 se espera que toda la oferta se encuentre disponible en este canal, ya que por el momento está desarrollada en un $80 \%$ para personas naturales (García, 2019). Con la implementación de la banca digital, nacen servicios fáciles de adquirir en tan solo cinco minutos, con pocos pasos, con beneficios en tasas y precios y disponibles las 24 horas del día, los 7 días de la semana.

A pesar de todas las inversiones y todos los esfuerzos realizados, solo el $60 \%$ de los clientes activos del Banco Amigo ingresan a los canales digitales para realizar consultas, transacciones y solicitudes financieras en su vida cotidiana (Banco Amigo, 2019a). El resto de los usuarios siguen haciendo sus operaciones en las oficinas de servicio, pese al esfuerzo ejecutado por la entidad para el fortalecimiento de la banca móvil. La compañía se ha trazado como meta que el $88 \%$ de sus clientes activos ingresen a la app o web, sin embargo, la tendencia muestra que esto no se logrará cumplir en el corto plazo con el crecimiento inercial dado hasta 2019 (Banco Amigo, 2019b).

A pesar de la acelerada transformación digital y todos los beneficios mencionados, la compañía no ha encontrado un plan para lograr que los usuarios conciban la importancia del uso de los productos disponibles en la app. La estrategia de marketing acogida por la entidad para promover las ventajas de los canales virtuales no ha tenido éxito. Intentan llegar a toda la población con el mismo mensaje a través de comunicaciones masivas, lo que ha convertido 
la herramienta del correo electrónico en un mundo infinito de correos basura, saturando a los actores del mercado que no tienen la disposición para leer cosas que no van en la misma dirección que los cambios que esperan tener. Actualmente, solo el $13 \%$ de los correos enviados son abiertos por los usuarios y, peor aún, solo el 0,5\% ejecuta la acción deseada (Banco Amigo, 2019c; 2019d).

La transformación digital continúa avanzando a pasos agigantados. No obstante, la adopción progresa de forma lenta al no haberse realizado un trabajo de manera correcta para lograr involucrar a los clientes en esta cultura. Es el momento de enfocar esfuerzos, hallar las palancas e incentivos necesarios para promover el uso de la banca móvil, identificando las objeciones y hábitos de consumo que tienen las personas, diseñando campañas personalizadas que aumenten la afiliación en cada segmento que se estructure, para así hacer frente a la amenaza latente de los nuevos jugadores en el sistema. Por ello, la contribución de los resultados de la investigación realizada en el presente trabajo juega un papel de primer nivel en la compañía.

\section{Metodología}

El trabajo se realizó en dos fases: en primera instancia, se hizo una investigación para lograr tener un análisis del entorno y encontrar las barreras y limitaciones que tienen los clientes para la adopción digital de los desarrollos del Banco Amigo. En segundo lugar, basados en los resultados de la investigación, se llevó a cabo la etapa de construcción de un modelo analítico para tener la base para la creación de un plan de adopción digital con estrategias personalizadas en cada uno de los segmentos de clientes. A través del modelo, se pudo obtener una estructura de costos por canal y tipo de cliente, lo que permitió realizar un detallado caso de negocio para cada una de las tácticas planteadas en la solución.

\section{Investigación}

\section{Análisis del entorno}

A través de fuentes secundarias se estudió el contexto de la infraestructura de las comunicaciones y las cifras de la industria para entender si las condiciones del mercado estaban dadas para adoptar la transformación digital impulsada por el Banco Amigo.

\section{Encuestas}

Se realizaron encuestas virtuales con preguntas cerradas con el objetivo de entender a profundidad las barreras para adoptar los canales digitales del Banco. Con el ejercicio, se identificaron las razones que tienen los clientes para preferir los canales físicos, y qué limitaciones tienen para realizar transacciones mediante la app móvil o la página web. 
El estudio se llevó a cabo tomando una muestra representativa para realizar el análisis cuantitativo. Las encuestas virtuales se hicieron a aquellos clientes que no usan los canales digitales y se encuentran activos dentro del Banco.

La estructura de la encuesta tenía 5 grandes categorías:

- Perfil de los encuestados: datos demográficos y ocupación.

- Conocimiento de canales digitales: se determina si los clientes han escuchado acerca del tema e identifican estos canales.

- Evaluación de los canales digitales del Banco Amigo, de acuerdo con las ventajas y desventajas que se perciben de los canales virtuales.

- Limitaciones y detonantes del no uso de los canales digitales: se identifican las razones por las cuales los clientes no ingresan a los canales digitales, y se trata de entender por qué razones es mayor el uso y la preferencia por las oficinas de servicio.

- Expectativas que tienen para acceder a la app o web del banco: medios esperados para recibir información y estrategias que esperan.

A través de la encuesta, se identificaron las barreras bajo tres enfoques específicos que tienen los clientes para adoptar la cultura que desea incentivar el Banco en sus usuarios:

- Factores personales: actitud relacionada con la disposición hacia lo nuevo, respecto de invertir tiempo, esfuerzo y energía en salir de la zona de confort. Aparecen respuestas a los cuestionamientos tipo: ¿están los clientes preparados para la adopción digital?, ¿tienen habilidades y competencias?, ¿tienen el apoyo de los demás?, ¿están en condiciones de hacerlo?, ¿los clientes conocen los beneficios?, ¿qué ganan en los canales digitales?

- Factores internos asociados a la oferta del Banco Amigo: percepción que tienen los clientes sobre los cobros que se hacen en los canales virtuales, capacitación del personal para acompañamiento, canales y comunicaciones y proceso de activación e ingreso.

- Factores externos, condiciones de los clientes para no poder acceder a los canales virtuales: recursos requeridos y situación sociodemográfica.

La población, de acuerdo con el objetivo de la investigación, era de 1500000 clientes, lo que corresponde a aquellos clientes activos que no ingresan a los canales digitales. Se construyó la base con los datos de contacto, se envió correo electrónico con la invitación a contestar la encuesta por medio de los canales oficiales del banco con acompañamiento de 
una firma dedicada a la investigación de mercados. Para el estudio se definió un nivel de confianza de 1,96, ya que es el más utilizado en un muestreo probabilístico.

La muestra constituye el segmento de la población que aportó la información necesaria para el desarrollo del estudio. El tamaño de la muestra representativa se obtuvo de la siguiente fórmula:

$$
n=\frac{Z^{2} * P * q * N}{\left(E^{2} * N\right)+\left(Z^{2} * P * q\right)}
$$

Donde:

$n$ : tamaño de la muestra.

$N$ : tamaño de la población.

$Z$ : nivel de confianza deseado. Para el caso, el valor de acuerdo con el criterio de la investigación es de 1,96 , equivalente a $95 \%$ de confianza.

$P$ : factor de aceptación de la muestra. Para el caso, $50 \%(0,50)$.

$q$ : factor de rechazo de la muestra. Para el caso, $50 \%(0,50)$.

$E$ : porcentaje de error admisible para la muestra. Para el caso, $5 \%(0,05)$.

En el caso de la presente investigación, la muestra era de 384 encuestados. Dadas las cifras de respuesta que se tienen en el banco, se enviaron correos a 7000 clientes y se tuvieron un total de 485 respuestas, pero 45 de ellas se encontraban incompletas, por lo que el análisis se realizó sobre 440.

\section{Creación del plan de adopción digital}

Para poder concretar estrategias que fueran eficaces para el Banco Amigo, se realizó un levantamiento de información para identificar segmentos donde se tienen bajos índices de adopción y enfocar las estrategias de la manera adecuada. Se construyó un modelo analítico basado en la transaccionalidad de los clientes de los últimos 12 meses, almacenada en la data lake del banco. Se extrajeron los datos y las variables requeridas para la herramienta. Se pasó la información a Cloudera, software de Oracle que permite almacenar y organizar la información. Con la data estructurada, se realizó la visual del modelo en Power BI, que permite hacer todos los análisis a través de filtros y presenta los hallazgos mediante gráficas.

A través del modelo analítico se pudieron identificar las preferencias transacción-canal de los usuarios que se quieran estudiar. Así, la herramienta clasificó a los clientes en tres segmentos:

- Escéptico (físico): individuos que realizan la mayoría de sus transacciones en las oficinas de servicio u otros canales físicos, prefiriendo como tipo de interacción el contacto humano. 
- Análogo: individuos cuyo canal transaccional predominante son los ATM/Multiservicios, IVR, Puntos Ágiles, prefiriendo como tipo de interacción las máquinas.

- Digital: individuos cuyo canal transaccional preferente, mientras les sea posible, son los canales digitales, prefiriendo como tipo de interacción apps e internet.

Para hacer análisis exhaustivos y detallados, en el modelo se pueden hacer los siguientes filtros:

- Por productos.

- Por transacciones.

- Por clientes que no pertenezcan a la comunidad digital.

- Por segmento.

- Por demografía: rangos de edad y ciudad donde viven.

Con el modelo analítico se logró establecer el costo de cada segmento de clientes, esto, con el fin de hacer casos de negocio a cada una de las estrategias planteadas, así se determina la eficiencia generada por los resultados de adopción de cada una de ellas y la posible inversión que puede hacer el banco de acuerdo con la rentabilidad generada. El Departamento de Planeación del Banco Amigo facilitó los costos por operación de cada uno de los canales, información que se muestra en la tabla 1.

Tabla 1. Costos asociados a los canales

\begin{tabular}{|c|c|}
\hline \multicolumn{2}{|l|}{ Costo por canal } \\
\hline Oficina & $\$ 3745,90$ \\
\hline$A p p$ & $\$ 704,00$ \\
\hline Web & $\$ 666,90$ \\
\hline ATM & $\$ 816,10$ \\
\hline Datafonos & $\$ 104,00$ \\
\hline Corresponsal bancario & $\$ 925,70$ \\
\hline CRP & $\$ 1827,30$ \\
\hline
\end{tabular}

Fuente: Banco Amigo (2019e).

Se adicionó el costo de cada operación al modelo, con ello se halló el costo promedio anual por cliente de cada uno de los segmentos, tal como se muestra en la tabla 2. 
Tabla 2. Costo por segmento

\begin{tabular}{|c|c|c|c|}
\hline Segmento & $\begin{array}{c}\text { Transacciones promedio por } \\
\text { cliente }\end{array}$ & $\begin{array}{l}\text { Costo promedio } x \\
\text { transacción }\end{array}$ & $\begin{array}{l}\text { Costo promedio por } \\
\text { cliente }\end{array}$ \\
\hline Digital & 163 & $\$ 804$ & $\$ 131074$ \\
\hline Análogo & 39 & $\$ 786$ & $\$ 30667$ \\
\hline Escéptico & 53 & $\$ 3.193$ & $\$ 169227$ \\
\hline
\end{tabular}

Fuente: Banco Amigo (2019f).

Con toda la información estructurada y los resultados del modelo analítico, se pasó a la construcción de las estrategias evaluando el impacto en los índices de adopción y la eficiencia en los costos generados para el Banco Amigo.

\section{Resultados e implicaciones}

\section{Análisis del entorno}

Para analizar si el mercado tiene las condiciones adecuadas para adoptar los desarrollos digitales, es necesario evaluar la infraestructura de telecomunicaciones. Este es el pilar para que los usuarios tengan acceso a los nuevos productos y funcionalidades que se vienen implementando en el sector financiero, a través de los canales digitales (app y web), priorizando la estrategia de tener todo en el celular del cliente.

Durante los últimos años, la inserción móvil en América Latina ha crecido a pasos agigantados, por ejemplo, GSMA (2019) — que es la entidad que representa los intereses de los operadores de telefonía móvil de todo el mundo, une a cerca de 750 operadores y a casi 400 empresas en el más amplio ecosistema de la telefonía móvil-calculó que en 2019 había 422 millones de suscriptores móviles únicos en toda América Latina, que representaban el $67 \%$ del total de la población.

Aproximadamente, el $80 \%$ de todos los suscriptores móviles también están suscritos a internet móvil, y se prevé que esta cifra aumente al $87 \%$ para 2025.

Con el impulso de la continua inversión en la red y las inminentes asignaciones de espectro, se acelerará la adopción de 4G en América Latina en los próximos años, alcanzando un 67 \% para 2025. Esto preparará el terreno para la tecnología 5G. Las dos tecnologías coexistirán durante mucho tiempo, de hecho, se espera que el primer lanzamiento comercial de $5 \mathrm{G}$ en la región sea en 2020 (en México, Uruguay y Brasil). Se prevé que América Latina llegue a una adopción de $5 \mathrm{G}$ del $7 \%$ para 2025. Las velocidades de datos más altas mejorarán la productividad en sectores que dependen de comunicaciones masivas. Por lo tanto, las empresas de la región consideran que la capacidad más importante de la tecnología 5G es la mejora en las velocidades de datos (OCDE, 2019). 
La situación en Colombia no difiere mucho del resto de la región. En materia de infraestructura de telecomunicaciones, existe un ambiente propicio para la adopción de la vía digital, en consecuencia, se eliminan las barreras tecnológicas para implementar un plan que impulse el uso de los canales virtuales. Según cifras del Ministerio de Tecnologías de la Información y Comunicaciones de Colombia (Mintic, 2019), el número de abonados en el servicio de telefonía móvil en Colombia alcanzó un total de 62822 720, es decir, un índice de penetración del 126,1 \% sobre el total de la población en Colombia. Del total de abonados, el $70 \%$ tiene un celular inteligente (smartphone) y el $61 \%$ de la sociedad tiene acceso de conexión a internet banda ancha, ya sea de modo fijo o móvil (Mintic, 2019).

El Mintic (2019) señala que, en cuanto acceso a internet móvil, al cierre del tercer trimestre de 2018, el número total de accesos por suscripción a internet fue de 11223377 suscriptores móviles (una participación de $63 \%$ ); mientras que los accesos fijos a internet alcanzaron los 6657339 suscriptores, con una participación de $37 \%$.

Todo lo anterior demuestra que las circunstancias están dadas para que los canales virtuales asuman el protagonismo que merecen, se tiene un avance significativo en la construcción de la banca móvil y los habitantes disfrutan de una buena penetración en telefonía e internet. Ahora es el momento de edificar un plan de adopción que comience a cambiar la cultura, generar valor y confianza, y que motive el uso de los canales digitales mediante incentivos y soluciones personalizadas.

\section{Hallazgos de las encuestas}

\section{Conocimiento de los canales digitales}

- Los encuestados de ciudades no capitales, que en su mayoría pertenecen a estratos 1 y 2 y que están conformados, principalmente, por amas de casa o empleados de servicios varios, asistentes u operativos, no han escuchado acerca de los canales virtuales y, por consiguiente, no los conocen.

- Los siguientes estratos, es decir: 3, 4, 5 y 6, han escuchado de los canales virtuales, pero no saben con certeza cuáles son.

- Las personas mayores de 55 años y los participantes de ciudades capitales con cargos asistentes, operativos y de servicios varios de estratos 1 y 2 , tienen un desconocimiento total de los canales virtuales.

- Se muestra una tendencia a que empleados e independientes que viven en Bogotá en estratos 3, 4, 5 o 6 han escuchado y conocen cuáles son los canales virtuales. 


\section{Limitaciones y detonantes del no uso de los canales digitales}

Entre el $65 \%$ y el $80 \%$ de los encuestados están totalmente de acuerdo con las siguientes afirmaciones:

- Creen no tener las habilidades para usarlos.

- Consideran que no son seguros.

- Piensan que si cometen un error el banco no se va a responsabilizar por este.

- Desconocen las acciones o funciones que pueden realizar.

- Consideran que los canales fueron hechos para otro tipo de personas

El $45 \%$ de los encuestados consideran que por tener pocos productos — en la mayoría de los casos una cuenta - no pueden hacer muchas acciones en los canales digitales.

El $38 \%$ de los clientes están totalmente de acuerdo en que no utilizan tales canales porque no cuentan con plan de datos y/o internet que les pueda servir en el caso de encontrarse en algún lugar cercano que visiten.

El $25 \%$ manifiesta estar de acuerdo o totalmente de acuerdo en que existen cobros adicionales.

\section{Factores emocionales, internos y externos asociados a los canales digitales}

Factores emocionales:

- Los clientes sienten no estar preparados para ingresar y hacer uso de esta tecnología.

- Perciben beneficios que brindan los canales digitales.

- Se destaca la inseguridad que sienten en el caso de utilizar la app o la web.

Factores internos:

- No hay claridad acerca de los beneficios que otorga el banco en tasas y costos por el uso de canales digitales.

- La comunicación no ha generado recordación: hay segmentos que no han escuchado, ni conocen cuáles son los canales virtuales.

- Los clientes tienen la expectativa y necesidad de recibir atención personalizada del paso a paso para utilizar los canales.

- Se considera que al tener pocos productos financieros no pueden hacer uso de la app o web.

Factor externo: 
- La situación sociodemográfica y económica es un factor limitante, los clientes que han adoptado los canales digitales tienden a ser de estratos 1 y 2 , residentes de ciudades no capitales con desconocimiento de estas tendencias tecnológicas y bajo presupuesto para pago de internet o plan de datos.

\section{Hallazgos del modelo analítico}

Con los análisis hechos en la herramienta se logran los siguientes hallazgos:

- $\quad$ El $60 \%$ de las transacciones son realizadas por clientes digitales.

- El $55 \%$ de las transacciones son digitales.

- Si los clientes escépticos hicieran sus transacciones en canales digitales, le costarían al banco \$36 233, generando una eficiencia de \$132994 por cliente.

- Si los clientes digitales hicieran sus transacciones en los canales físicos, su costo sería de $\$ 558918$, en vez de los $\$ 131074$ que le cuestan actualmente al banco.

\section{Plan para aumentar los índices de adopción digital}

Con la investigación, se identifica que la cantidad de correos no personalizados que ha utilizado el banco para comunicar sus avances en transformación digital trae consigo la omisión de mensajes. Para ser efectivos, es primordial estructurar un plan que entienda los ciclos de vida y necesidades de cada segmento de clientes en particular.

Con el crecimiento de los procesos analíticos como Big Data y Data Analytics, utilizados para la creación del modelo analítico y para pronosticar comportamientos, el Banco está en la capacidad de predecir y anticiparse al comportamiento de los clientes; de este modo, la adopción de la vía digital provee una solución fácil y sencilla a los requerimientos puntuales que tiene cada uno de los individuos del mercado. Los consumidores esperan que les hablen en su mismo lenguaje, de forma clara y concreta, pues quieren escuchar un relato acorde a los sueños que desean cumplir.

Los correos y SMS diseñados por el banco son tradicionales y racionales, muestran los beneficios del producto y sus respectivos cobros y tasas. Pero ya es hora de una transformación, tal y como señala Godin (2019), las fuerzas irracionales son las que impulsan a los clientes, lo que esté ligado a sus emociones va a generar un mayor impacto. Es preciso elaborar estrategias que forjen felicidad, estatus y afinidad.

Para superar las limitaciones que afectan el uso de los canales digitales según los resultados de las encuestas, se estructuraron estrategias afines a lo que esperan los usuarios, evaluando el impacto en las metas del banco y el esfuerzo requerido para la implementación en términos 
de tiempo y costo. A este respecto, a continuación, se detalla cada una de las estrategias que componen el plan.

\section{Consumo datos asumidos por el banco}

Al estudiar a los encuestados que manifiestan que la limitante para acceder a los canales digitales es que requieren plan de datos en su celular, se encuentra que son personas de cargos operativos con un salario inferior o igual a 2 SMMLV. En el Banco se clasifica a este segmento de usuarios como inclusión. A través del login para ingresar a los canales digitales se reconoce a los clientes a través de su tipo de identificación y número de identificación, así se puede determinar quiénes pertenecen al segmento de inclusión. Ellos no necesitarán tener plan de datos para ingresos a la app, ya que el banco asumirá el costo de este consumo.

\section{Centros de experiencias digitales para adultos mayores}

Con los resultados de la investigación se percibe que los clientes se sienten ignorantes frente al tema de los canales digitales, pueden tener miedo a preguntar sobre su uso porque suponen que no les tienen paciencia y que los consideran poco inteligentes. Ante esto, es el momento para generar un ambiente de confianza con el fin de que los clientes se animen al cambio y puedan dedicar tiempo a entender los beneficios y funcionalidades de los canales digitales.

Mediante el modelo analítico se encontró que el $75 \%$ de los clientes activos que ingresan a los canales digitales están entre los veinticinco y cincuenta y cinco años. La generación es un factor importante en la adopción digital, hay un reto esencial en los baby boomers, personas que requieren y demandan su aprendizaje de forma diferente a como se viene abordando en el banco.

Actualmente, el promedio de edad de los funcionarios de oficinas del banco es de 32 años (Banco Amigo, 2019g). Son jóvenes familiarizados con la tecnología, pero los clientes mayores no los tan ven cercanos como para que puedan atender sus necesidades con respecto al uso de los canales virtuales. Es necesario cambiar la dinámica de la entidad para conseguir resultados en este importante nicho de mercado, asimismo, se deben crear centros de experiencias atendidos por adultos mayores expertos digitales, que manejen el mismo idioma y conceptos de la población a la que se quiere atender.

Con este cambio, los adultos mayores se sentirán en plena confianza, con unas oficinas creadas para ellos, con atención personalizada a través de su mismo círculo social y así podrán compartir experiencias de lo aprendido con sus amigos dentro de estas instalaciones. La idea de la estrategia es que los baby boomers conozcan y entiendan todos los beneficios de los canales digitales, accediendo desde canales físicos con un adecuado acompañamiento. En estos centros de experiencia se ofrecerán desayunos, charlas, cata de vinos para que el ambiente sea más propenso a que se disfrute el tiempo libre y, a la vez, aprendan. Esta 
estrategia va de la mano con otro objetivo al que ha apuntado la empresa para el 2020: ser el banco de los pensionados y tener prácticas enriquecedoras para este segmento.

Esta arista del plan de adopción está alineada con las tendencias de omnicanalidad. Empresas como Google y Amazon han enriquecido su experiencia de compra digital con centros de servicios que brindan y emulan la experiencia brindada en los canales digitales. Así, el cliente puede interactuar entre apps, web y oficinas físicas, sin ser canales independientes, sino que se complementan entre sí.

Se está buscando la forma de unificar la experiencia en los canales en los que está presente el negocio. Hoy, el banco solo tiene implementada la multicanalidad, donde la relación con el cliente, pese a darse a través de todos los canales en los que la empresa está presente, comienza y termina en el mismo canal, no hay convergencia entre ellos.

\section{Charlas de educación financiera en seguridad de los canales digitales}

El banco ha construido muchas herramientas para blindar las transacciones de los clientes en los canales digitales, sin embargo, no son conocidas en el mercado. Por ello, a través del Departamento de Educación Financiera del Banco Amigo, se desea fabricar un programa de capacitación que contenga los instrumentos de seguridad que se han desarrollado en los canales virtuales del banco. El programa tendrá el siguiente alcance:

- Reconocimiento facial.

- Reconocimiento dactilar o biometría.

- Reconocimiento de documentos de identidad.

- Software de seguridad que protege sus datos e información financiera.

- Sistemas de autenticación y notificaciones (claves o códigos de confirmación).

- Registro de dispositivos.

- Trazabilidad de las operaciones y los recursos, hoy es posible hacer pleno seguimiento sobre qué pasa con cada transacción electrónica para identificar el origen del dinero, su destino, la cantidad y el tiempo exacto de su realización. Esto hace que cada vez sea más difícil que se presenten fraudes.

Para tener un impacto significativo con el material construido, la estrategia se llevará a cabo por medio de charlas a los empleados de nómina. Se visitarán las empresas que tienen convenio de nómina con el banco, para compartir un material didáctico con el fin de que los empleados conozcan, de primera mano, cómo la entidad protege su dinero cuando realizan operaciones y consultas en los canales virtuales. 
Para cubrir otro segmento importante en este ítem, se llegará a los pensionados por medio de los eventos realizados en todo el país por Colpensiones, las Fuerzas Militares y los fondos privados, donde el Banco Amigo es patrocinador oficial.

Se toman estos dos segmentos por ser focos estratégicos fundamentales para la adopción digital, ya que se garantiza que son clientes con cuentas de ahorro que reciben dinero a través de la nómina y la mesada pensional, por consiguiente, son usuarios que necesitan transar con los productos del Banco, ya sea para realizar transferencias o pagos, entre otros.

\section{Campañas personalizadas en tiempo real}

Los clientes encuestados se encuentran activos dentro del banco, eso quiere decir que, si no están haciendo consultas y operaciones a través de los canales digitales, los están realizando en las oficinas, sin importar la cantidad de productos que posean. Cabe recordar que la oferta de canales virtuales está diseñada inclusive para usuarios que cuentan con solo un producto. Los clientes llegan a pensar que los canales virtuales no están construidos para ellos, porque los correos masivos que envía el banco muestran todas las funcionalidades que tiene la aplicación, pero nunca le hablan al usuario de manera personalizada, resaltando lo que está haciendo exactamente en la oficina como lo podría hacer en su celular, sin desplazamientos, sin costos adicionales y de manera fácil y rápida.

El modelo analítico juega un papel fundamental en esta estrategia, porque muestra exactamente las operaciones que cada grupo de clientes está haciendo en los canales físicos. Los pasos a seguir para convertir esta información en campañas realmente efectivas que logren mejorar los índices de adopción digital son los siguientes:

- Realizar un desarrollo para conectar el modelo analítico a un software que compró el Banco, llamado RTD (Real Time Decision), que permite enviar campañas en tiempo real.

- Con la conexión del modelo analítico al software, se deben parametrizar los triggers que originan las campañas.

- Una vez se origina la campaña, se otorga una comunicación personalizada, interactiva y con acompañamiento. Automáticamente le llega una llamada al cliente para explicar cómo puede realizar la transacción que esté haciendo en oficina, pero dentro de los canales digitales. Por ejemplo, si el cliente en la oficina del banco realiza una transferencia de su cuenta hacia la de un tercero, inmediatamente se dispara una llamada de un BPO (Business Process Outsourcing) contratado en el banco para realizar la acción con el usuario paso a paso. 


\section{Mejorar UX/UI de procesos específicos}

Actualmente, la app del banco mantiene una única fuente de letra, no se configura en simultáneo cuando el cliente cambia el tamaño en los ajustes generales de su celular. Por ello, se requiere realizar un desarrollo para tener diseños responsive, para que una vez se ordene el tamaño de la letra en los ajustes del celular la app también lo haga de manera automática.

Por otro lado, es indispensable implementar el proyecto de clave única para todos los canales del Banco: esto va muy alineado con la omnicanalidad comentada anteriormente. Hoy la compañía tiene las siguientes claves como mecanismos de autenticación y confirmación:

- Clave tarjeta débito para retiros en cajeros.

- Clave de avances de tarjetas de crédito.

- Clave virtual para ingreso a los canales digitales.

- Códigos de confirmación para transacciones en canales digitales.

Además, en el proceso de asignación de la clave virtual se piden los últimos 4 números de alguno de los productos, una clave que tenga activa (puede ser la de la tarjeta débito) y luego se finaliza con el registro. Ante tantas solicitudes de números el cliente queda perdido.

Lo que se busca hacer es que solo se tenga un proceso de asignación de clave, por el canal que el cliente desee, y que dicha clave se convierta en la puerta de entrada para todos los servicios del banco.

\section{Impacto de las estrategias}

A continuación, en la tabla 3, se presenta el resumen de todas las estrategias planteadas en el presente documento:

Tabla 3. Resumen de estrategias

\begin{tabular}{llllll}
\multicolumn{1}{|c}{ Estrategia } & $\begin{array}{c}\text { Impacto en } \\
\text { cantidad de } \\
\text { clientes }\end{array}$ & $\begin{array}{c}\text { Tiempo de } \\
\text { implementación } \\
\text { (meses) }\end{array}$ & $\begin{array}{c}\text { Costo de } \\
\text { implementación } \\
\text { (MCOP) }\end{array}$ & $\begin{array}{c}\text { Eficiencia en } \\
\text { costos (MCOP) }\end{array}$ & $\begin{array}{c}\text { Ganancia neta } \\
\text { (MCOP) }\end{array}$ \\
$\begin{array}{l}\text { 1. Consumo } \\
\text { datos asumidos } \\
\text { por banco. }\end{array}$ & 110000 & 5 & $\$ 798$ & $\$ 52665$ & $\$ 51867$ \\
\hline $\begin{array}{l}\text { 2. Centros de } \\
\text { experiencias } \\
\text { digitales para } \\
\text { adultos mayores. }\end{array}$ & 150000 & 8 & $\$ 155400$ & $\$ 172320$ & $\$ 16920$ \\
\hline
\end{tabular}




\begin{tabular}{|c|c|c|c|c|c|}
\hline Estrategia & $\begin{array}{l}\text { Impacto en } \\
\text { cantidad de } \\
\text { clientes }\end{array}$ & $\begin{array}{c}\text { Tiempo de } \\
\text { implementación } \\
\text { (meses) }\end{array}$ & $\begin{array}{c}\text { Costo de } \\
\text { implementación } \\
(\mathrm{MCOP})\end{array}$ & $\begin{array}{c}\text { Eficiencia en } \\
\text { costos (MCOP) }\end{array}$ & $\begin{array}{l}\text { Ganancia neta } \\
\text { (MCOP) }\end{array}$ \\
\hline $\begin{array}{l}\text { 3. Charlas de } \\
\text { educación } \\
\text { financiera en } \\
\text { seguridad de los } \\
\text { canales digitales. }\end{array}$ & 147000 & 9 & $\$ 20095$ & $\$ 35634$ & $\$ 15538$ \\
\hline $\begin{array}{l}\text { 4. Campañas } \\
\text { personalizadas } \\
\text { en tiempo real. }\end{array}$ & 211000 & 8 & $\$ 10111$ & $\$ 60512$ & $\$ 50401$ \\
\hline $\begin{array}{l}\text { 5. Mejorar } \\
\text { UX/UI de } \\
\text { procesos } \\
\text { específicos. }\end{array}$ & 285000 & 12 & $\$ 5790$ & $\$ 37903$ & $\$ 32113$ \\
\hline Total & 903000 & & \$ 192194 & $\$ 359036$ & $\$ 166841$ \\
\hline
\end{tabular}

\section{Conclusiones}

Como se ha evidenciado a lo largo de este artículo, hay una tendencia mundial en lo que se refiere a la implementación de la digitalización de servicios; en este sentido, el sector financiero es uno de los que está sufriendo mayores transformaciones. El surgimiento de las fintech ha aportado bastante a la evolución hacia las soluciones vía celular, por tal razón, es imprescindible que se adopte este camino para garantizar la permanencia en el mercado en unos años.

Dadas las circunstancias, el Banco Amigo ha emprendido en Colombia un proyecto ambicioso de transformación digital, invirtiendo en el desarrollo de la banca móvil para potencializar su oferta. Sin embargo, la adopción de los canales digitales presenta un ritmo de crecimiento más lento.

A través de las encuestas fue posible identificar las barreras que poseen los clientes para adoptar los desarrollos realizados por el Banco Amigo en los canales digitales. De allí se obtuvo como conclusión que no se ha dado el acompañamiento adecuado para lograr una cultura digital donde los usuarios disfruten los beneficios de la banca móvil, por ello, se encuentran confusos y con desconfianza frente a sus ventajas y su uso.

Luego de estudiar los resultados de las encuestas, se hizo necesario construir un modelo analítico que permitiese conocer cómo los clientes interactúan con los canales del banco, con el fin de lograr llegar de manera personalizada a lo que cada grupo requiere.

Con los insumos presentados en la investigación, se edificaron estrategias que constituyen el plan de adopción digital con el cual se atienden los impedimentos manifestados por los clientes. A través de las propuestas se dan resultados concretos al vincular 903000 usuarios 
a los canales virtuales, así la compañía tendría al $84 \%$ de sus clientes activos realizando consultas y transacciones por medio de su banca móvil. Se pasa del $60 \%$ al $84 \%$ de clientes digitales, dando un impulso considerable para llegar al resultado esperado por el banco, que consiste en alcanzar un $88 \%$ de clientes ingresando a los canales virtuales.

Lo más importante del resultado son las eficiencias que genera el banco en las implementaciones, lo que significa una recuperación del valor invertido en el corto plazo. Por cada cliente que adopte los canales digitales se logra un $75 \%$ de eficiencia en costos, el costo total de las estrategias es de 192194 millones de pesos y las deficiencias percibidas 359036 millones de pesos, logrando una ganancia neta de 166841 millones de pesos.

Con base en lo anteriormente expuesto se dan los siguientes avances para la compañía:

- Para sus clientes: tendrán campañas personalizadas de beneficios a los que pueden acceder de manera rápida y sencilla mediante el uso de los canales digitales, solucionando necesidades específicas de su vida cotidiana, metas y sueños. Los usuarios no se verán exhaustos y molestos por ofertas que hoy reciben y no tienen lugar.

- Para el Banco Amigo: sus esfuerzos e inversiones destinados a la transformación digital no se verán despreciados, este trabajo ofrece la solución que impulsa la adopción de los desarrollos y cambios tecnológicos implementados. Ahora el banco conoce de manera detallada a sus clientes y lo que esperan, para enfocar sus estrategias en pro de un mejor relacionamiento logrando así:

- Aumentar el porcentaje de usuarios en los canales virtuales que realizan transacciones y solicitudes de productos.

- Eficiencia en los costos de los canales transaccionales al tener clientes digitales que priorizan hacer sus transacciones y solicitudes de productos a través de la banca digital.

- Incrementar sus ventas de productos: los clientes recibirán ofertas acordes a sus necesidades puntuales, aumentando la efectividad de las campañas de marketing.

○ Incrementar su seguridad: con la adopción digital se disminuye el manejo del efectivo, por consiguiente, se presentan menos posibilidades de robo. La empresa podrá robustecer sus plataformas de control y auditorías basadas en el conocimiento transaccional del cliente, para monitorear sus operaciones y prevenir de manera proactiva los fraudes. Lo anterior repercute directamente en la satisfacción de los usuarios; como se mostró en la investigación una de sus principales preocupaciones es que se sienten intranquilos al manejar sus finanzas desde los canales virtuales. 


\section{C̈IFFEิ 38}

- Contribuir a problemáticas legales y sociales del país: por medio de la banca móvil se puede llegar a tener una detallada trazabilidad del dinero, evitando lavado de activos y evasión de impuestos.

- Preservar el medioambiente: en las solicitudes de productos y transacciones digitales no se exigen papeles, esto repercute en la tendencia mundial de cuidar el planeta y es congruente con la estrategia de la entidad de 'cero papeles', que le permite mantenerse vigente en el índice de sostenibilidad del Dow Jones, que valora las buenas prácticas de las empresas bajo criterios sociales, medioambientales y económicos.

Adicional a todo lo planteado, hay una huella social detrás la adopción que tendrán los clientes de estas nuevas tecnologías. Con el acceso a los canales virtuales el Banco Amigo se convierte en un promotor fundamental en la bancarización y profundización de los clientes, los usuarios no van a necesitar documentos para solicitar los productos y al tener mejores tasas y cobros pueden estar en la capacidad de pagar un valor asequible para dar solución a su situación financiera del momento, logrando cumplir con metas específicas como tener un vehículo y un techo para su hogar. Es aquí donde la adopción digital toma su mayor relevancia, pues está demostrado que tener más oportunidades de alcanzar servicios bancarios repercute en la calidad de vida y bienestar de un país, contribuyendo además a la reducción de la pobreza.

Un estudio realizado en Nepal, por ejemplo, demostró que el acceso de su población a cuentas y créditos a través de canales virtuales aumentó la inversión de los hogares en educación, disminuyó la desigualdad de ingresos, forjó la creación de empleos formales y aumentó la capacidad de ahorro de las personas para reducir su vulnerabilidad ante las crisis (Banca de las oportunidades y Superintendencia Financiera de Colombia, 2016).

\section{Referencias}

Banca de las oportunidades y Superintendencia Financiera de Colombia. (2016). Reporte de Inclusión Financiera Banca de las Oportunidades. http://bancadelasoportunidades.gov.co/sites/default/files/201807/RIF \%202016- \%20final.pdf

Banco Amigo. (2019a). Detalle transaccionalidad monetaria y no monetaria por cliente en canales digitales. Proveedor. BI Proveedor Banco Amigo.

Banco Amigo. (2019b). Informe efectividades campañas comunicaciones dirigidas. Departamento Publicidad. Drive campañas Banco Amigo.

Banco Amigo. (2019c). Informe mensual análisis transaccional y demográfico de clientes. Departamento Analítica de Clientes Plataforma BI Banco Amigo. 


\section{CIIFF 38}

Banco Amigo. (2019d). Informe mensual comunidad digital. Proveedor. BI Proveedor Banco Amigo.

Banco Amigo. (2019e). Presupuesto y metas adopción digital 2020. Dirección Movilidad y Canales. Drive Presentación presupuestos presidencia 2020.

Banco Amigo. (2019f). Resultados anuales estrategia digital. Departamento Planeación Financiera. Tablero de control Alta Gerencia Banco Amigo.

Banco Amigo. (2019g). Resultados trimestrales PyG productos y transacciones. Departamento Planeación Financiera. Tablero de control Banco Amigo.

Banco Mundial (2019). Informe de inclusión financiera en América Latina. http://www.bancomundial.org/

Foro Económico Mundial. (13 de marzo de 2016). América Latina en un punto de inflexión: construyendo una nueva narrativa. http://www3.weforum.org/docs/LA18_Report_Spanish.pdf

García, A. (12 de mayo de 2019). "Para 2020, todos los bancos serán digitales": Davivienda. El Tiempo. https://www.eltiempo.com/economia/sectorfinanciero/presidente-de-davivienda-dice-que-en-el-2020-todos-los-bancos-serandigitales-360634

Godin, S. (2019). Esto es marketing. Planeta.

GSMA Association. (2019). La economía móvil en América Latina 2019. gsma Association.

Heredia, R. (2019). Ecosistemas digitales: La revolución de todas las industrias. Una oportunidad para la industria financiera Latinoamericana. Digital Bank Latam, Zyos y Escuela de Innovación.

Ministerio de Tecnologías de la Información y las Comunicaciones (Mintic). (2019). Boletín trimestral de las TIC: Cifras tercer trimestre de 2018. https://colombiatic.mintic.gov.co/679/articles-82350 archivo_pdf.pdf

OCDE. (2019). OECD Reviews of Digital Transformation: Going Digital in Colombia. OECD Publishing.

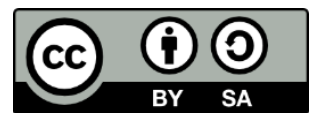

